\title{
Quantum correlations in one-dimensional Wigner molecules
}

\author{
Przemysław Kościk ${ }^{\mathrm{a}}$ \\ Institute of Physics, Jan Kochanowski University, ul. Świętokrzyska 15, 25-406 Kielce, Poland
}

\author{
Received 12 June 2017 / Received in final form 3 August 2017 \\ Published online 14 November 2017 \\ (C) The Author(s) 2017. This article is published with open access at Springerlink.com
}

\begin{abstract}
We studied one-dimensional systems formed by $N$ identical particles confined in a harmonic trap and subject to an inverse power-law interaction potential $\sim|x|^{-d}$. The correlation properties of a Wigner molecule with the lowest energy are investigated in terms of their dependence on the number $N$ and the power $d$, including the limit as $d \rightarrow 0$. There are $N$-particle Wigner molecules with properties such that their correlations are mainly manifested in the $N$ lowest natural orbitals. The values of the control parameters of the system at which such states appear are identified. The properties of Wigner molecules formed in the limit as $d$ approaches infinity are also revealed.
\end{abstract}

\section{Introduction}

Soon after the birth of quantum mechanics, the study of quantum correlations began to attract significant research attention. In particular, the study of the many-body properties of various quantum composite systems is nowadays one of the most active areas of theoretical physics [1-23]. Investigating quantum correlations in systems of interacting particles trapped in external potentials is not only important in view of the context of quantum information technology [24] but also a key to improving our understanding of quantum matter. Various physical realizations of such systems are achieved nowadays. The advantage of artificially created systems is the possibility of controlling their properties, such as the number of particles, their interactions, and the shape of the trapping potential as well.

In addition to well-known ultracold atomic gases with short-range interactions, systems with long-range interactions have been created in laboratories. In particular, tremendous technological progress has been made over the last two decades and opened new perspectives for achieving the van der Waals and dipolar interactions in polar molecule experiments $[25,26]$. Other well-known examples of artificially created systems exhibiting long-range interactions are quantum dots $[27,28]$ and systems of electromagnetically trapped ions [29]. Such systems exhibit a variety of strongly correlated states. Among them is the famous Wigner phase [30], which manifests itself in the localisation of particles around their classical equilibrium positions.

The Wigner molecule-like states, formed by particles interacting via long-range interactions, have been widely studied in various theoretical contexts. For example, the

\footnotetext{
${ }^{\mathrm{a}}$ e-mail: koscik@pu.kielce.pl
}

studies include systems with interaction potentials such as the Coulomb potential [15-19], the inversely quadratic potential [20-22] and dipole-dipole interactions [16,23]. The Wigner molecules have also been observed in a variety experiments [29,31-34].

The task in the studies here is to carry out a comprehensive investigation of the correlation in the Wigner molecule's ground state, of the one-dimensional (1D) system

$$
\begin{gathered}
\mathcal{H}=-\frac{1}{2} \sum_{i=1}^{N} \frac{\partial^{2}}{\partial x_{i}^{2}}+\mathcal{V}\left(x_{1}, x_{2}, \ldots, x_{N}\right), \\
\mathcal{V}\left(x_{1}, x_{2}, \ldots, x_{N}\right)=\frac{1}{2} \sum_{i=1}^{N} x_{i}^{2}+\sum_{i>j=1}^{N} \frac{g}{\left|x_{i}-x_{j}\right|^{d}},
\end{gathered}
$$

which arises at positive values of $d$ in the large interaction strength regime, $g \gg 1$.

In our study, we use a scheme based on harmonic approximation (HA), developed in $[15,16]$, which enables an easy determination of various characteristics of the Wigner molecule state, such as the density, occupation numbers, and the entanglement measures. The HA yields exact results in the limit as $g \rightarrow \infty(d>0)[16,18,19,21]$. However, this fails strictly within the limit as $d \rightarrow \infty$, i.e., when the interaction potential becomes the hard-core potential [35].

The structure of this paper is as follows. Section 2 outlines the formalism based on the HA to analyse the correlation properties of the Wigner molecule states. In addition, it provides an effective procedure for the analysis of the special case as $d \rightarrow 0$. Section 3 focuses on 
the question of how the changes in the parameter $d$ affect the correlation in the $N$-particle Wigner molecule groundstate. Finally, Section 4 presents concluding remarks.

\section{Methods}

The potential $\mathcal{V}$ given by equation (2) attains its minimum at $N$ ! points. Here we refer to the point $\vec{r}_{\text {min }}=$ $\left(x_{1}^{c}, x_{2}^{c}, \ldots, x_{N}^{c}\right)$ with $x_{1}^{c}<x_{2}^{c}<\cdots<x_{N}^{c}, x_{i}^{c}=-x_{N-i+1}^{c}$, $\left(x_{\frac{N+1}{2}}^{c}=0\right.$ if $N$ is odd), and denote it by $\vec{r}^{(0)}$. The equilibrium positions have the form $x_{i}^{c}=\beta_{i}^{c} g^{\frac{1}{2+d}}$, where $\beta_{i}^{c}$ satisfy the set of equations $\partial_{\beta_{k}} \mathcal{V}^{g=1}\left(\beta_{1}, \ldots, \beta_{N}\right)=0$,

$$
\mathcal{V}^{g=1}\left(\beta_{1}, \ldots, \beta_{N}\right)=\frac{1}{2} \sum_{i=1}^{N} \beta_{i}^{2}+\sum_{i>j=1}^{N} \frac{1}{\left|\beta_{i}-\beta_{j}\right|^{d}} .
$$

The values of $\beta_{i}$ are known analytically only for $N=2,3$ [18]. The exception is the limit as $d \rightarrow \infty$ in which $\beta_{i} \rightarrow$ $(2 i-N-1) / 2, i=1, \ldots, N$. It can be inferred that in the limit of small $d$ in which

$$
|x|^{-d} \rightarrow 1-d \ln |x|,
$$

the equilibrium positions of the particles $x_{i}^{c}$ tend to $x_{i}^{c}=$ $\alpha_{i}^{c} \sqrt{d g}$, where $\alpha_{i}^{c}$ are the solutions of the set of equations $\partial_{\alpha_{k}} \mathcal{V}^{d \rightarrow 0}\left(\alpha_{1}, \ldots, \alpha_{N}\right)=0$,

$$
\mathcal{V}^{d \rightarrow 0}\left(\alpha_{1}, \ldots, \alpha_{N}\right)=\sum_{i=1}^{N} \alpha_{i}^{2}-\sum_{i>j=1}^{N} \ln \left(\alpha_{i}-\alpha_{j}\right)^{2} .
$$

Within the HA the total potential (2) is approximated harmonically around $\vec{r}^{(0)}$,

$$
\mathcal{V}(\vec{r}) \approx \mathcal{V}\left(\vec{r}^{(0)}\right)+\frac{1}{2 !}\left(\vec{r}-\vec{r}^{(0)}\right)^{T} \mathbf{H}\left(\vec{r}-\vec{r}^{(0)}\right),
$$

where $\mathbf{H}$ is the so-called Hessian matrix, which can be expressed as [16]

$$
\mathbf{H}=\left[\left.\frac{\partial^{2} \mathcal{V}\left(\beta_{1}, \ldots, \beta_{N}\right)}{\partial \beta_{m} \partial \beta_{k}}\right|_{\left\{\beta_{i}=\beta_{i}^{c}\right\}}\right]_{N \times N} .
$$

In the particular case as $d \rightarrow 0$, we obtained

$$
\mathbf{H}^{d \rightarrow 0}=\frac{1}{2}\left[\left.\frac{\partial^{2} \mathcal{V}^{d \rightarrow 0}\left(\alpha_{1}, \ldots, \alpha_{N}\right)}{\partial \alpha_{m} \partial \alpha_{k}}\right|_{\left\{\alpha_{i}=\alpha_{i}^{c}\right\}}\right]_{N \times N},
$$

where $\mathcal{V}^{d \rightarrow 0}$ is given by (4).

It is well-known that under the approximation (5) the problem can be reduced to $N$ uncoupled oscillators [18]

$$
\mathcal{H}^{H A}=\sum_{i=1}^{N}-\frac{1}{2} \frac{d^{2}}{d q_{i}^{2}}+\frac{v_{i}^{2} q_{i}^{2}}{2},
$$

where the values of $v_{i}^{2}$ are the eigenvalues of $\mathbf{H}$. The coordinates $Q=\left(q_{1}, q_{2}, \ldots, q_{N}\right)^{T}$ (normal modes) are given by $Q=\mathbf{U} \overrightarrow{\mathbf{Z}}$, where $\overrightarrow{\mathbf{Z}}=\left(z_{1}, z_{2}, \ldots, z_{N}\right)^{T}, z_{i}=x_{i}-x_{i}^{c}$, and $\mathbf{U}$ is a matrix of eigenvectors of $\mathbf{H}$.

In terms of the HA, the asymptotic symmetric $(+)$ and antisymmetric (-) wavefunctions of a given energy level can be constructed as follows:

$$
\begin{aligned}
\Psi_{g \rightarrow \infty}^{( \pm)}\left(x_{1}, x_{2}, \ldots, x_{N}\right)= & \frac{1}{\sqrt{N !}} \sum_{p} A_{p} \psi_{\vec{n}}\left(x_{p(1)}-x_{1}^{c}, x_{p(2)}\right. \\
& \left.-x_{2}^{c}, \ldots, x_{p(N)}-x_{N}^{c}\right),
\end{aligned}
$$

where

$$
\begin{aligned}
\psi_{\vec{n}}\left(z_{1}, z_{2}, \ldots, z_{N}\right)= & \prod_{i=1}^{N}\left(\frac{v_{i}}{\pi}\right)^{\frac{1}{4}} \frac{1}{\sqrt{2^{n_{i}} n_{i} !}} \mathrm{e}^{-\frac{v_{i} q_{i}^{2}\left(z_{1}, z_{2}, \ldots, z_{N}\right)}{2}} \\
& \times H_{n_{i}}\left(\sqrt{v_{i}} q_{i}\left(z_{1}, z_{2}, \ldots, z_{N}\right)\right), \quad(10)
\end{aligned}
$$

is the corresponding eigenfunction of the Hamiltonian (8) and $H_{n}$ is the $n$th order Hermite polynomial. The sum in (9) is over all permutations, and $A_{p}$ is 1 for $(+)$. For $(-)$, $A_{p}$ is 1 for even permutations and -1 for odd permutations. In practise, the HA works well in the regime where the localised wave packets of the particles do not overlap much, i.e., when the Wigner molecule is formed. The general trend is that, the larger is the value of $d$, the larger is the value of $g$ at which the Wigner crystal behaviour is reached.

The integral representation of the asymptotic oneparticle reduced density matrix (1-RDM) has the form $[15,16]$

$$
\Gamma^{g \rightarrow \infty}\left(x, x^{\prime}\right)=\sum_{i=1}^{N} \rho_{i}\left(x, x^{\prime}\right),
$$

where the partial components $\rho_{i}$ are given by the following integrals

$$
\begin{aligned}
\rho_{i}\left(x, x^{\prime}\right)= & \frac{1}{N} \int_{\Re^{N-1}}\left(\prod_{k \neq i} d z_{k}\right) \\
& \times \psi_{\vec{n}}\left(z_{1}, \ldots, z_{i-1}, x-x_{i}^{c}, z_{i+1}, \ldots, z_{N}\right) \\
& \times \psi_{\vec{n}}\left(z_{1}, \ldots, z_{i-1}, x^{\prime}-x_{i}^{c}, z_{i+1}, \ldots, z_{N}\right) .
\end{aligned}
$$

The Wigner crystal ground-state wavefunction that we are interested in only corresponds to the lowest energy wavefunction of (8); that is,

$$
\psi\left(z_{1}, z_{2}, \ldots, z_{N}\right)=\prod_{i=1}^{N}\left(\frac{v_{i}}{\pi}\right)^{\frac{1}{4}} \mathrm{e}^{-\frac{v_{i} q_{i}^{2}\left(z_{1}, z_{2}, \ldots, z_{N}\right)}{2}} .
$$

In this case, the integrals (12) and the diagonal form of (11) can be obtained explicitly, namely [16],

$$
\rho_{i}\left(x, x^{\prime}\right)=A_{i} \mathrm{e}^{-a_{i}\left[\left(x-x_{i}^{c}\right)^{2}+\left(x^{\prime}-x_{i}^{c}\right)^{2}\right]+b_{i}\left(x-x_{i}^{c}\right)\left(x^{\prime}-x_{i}^{c}\right)},
$$




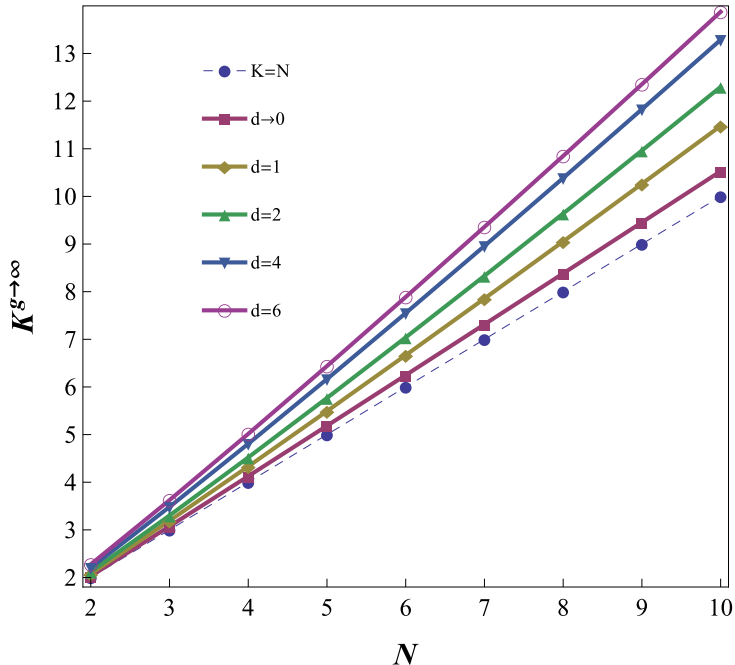

Fig. 1. The dependence of $\mathrm{K}^{g \rightarrow \infty}$ on $N$ for several values of $d$.

$a_{i}, b_{i}>0$ and

$$
\Gamma^{g \rightarrow \infty}\left(x, x^{\prime}\right)=\sum_{i=1}^{N} \sum_{l=0}^{\infty} \lambda_{l}^{(i)} u_{l}^{(i)}(x) u_{l}^{(i)}\left(x^{\prime}\right),
$$

respectively, where

$$
u_{l}^{(i)}(x)=\frac{\eta_{i}^{\frac{1}{4}}}{\pi^{\frac{1}{4}} \sqrt{2^{l} l !}} \mathrm{e}^{-\frac{1}{2} \eta_{i}\left(x-x_{i}^{c}\right)^{2}} H_{l}\left(\sqrt{\eta_{i}}\left(x-x_{i}^{c}\right)\right),
$$

and

$$
\lambda_{l}^{(i)}=A_{i} \sqrt{\frac{\pi\left(1-y_{i}^{2}\right)}{\eta_{i}}} y_{i}^{l},
$$

with

$$
\begin{gathered}
\eta_{i}=\sqrt{4 a_{i}^{2}-b_{i}^{2}}, \\
y_{i}=\frac{\sqrt{2 a_{i}+b_{i}}-\sqrt{2 a_{i}-b_{i}}}{\sqrt{2 a_{i}+b_{i}}+\sqrt{2 a_{i}-b_{i}}} .
\end{gathered}
$$

Note that $\left\langle u_{l}^{(i)} \mid u_{k}^{(i)}\right\rangle=\delta_{l k}$ and the integral overlap $\left\langle u_{l}^{(i)} \mid u_{k}^{(j)}\right\rangle$ vanishes for any $i \neq j$ in the limit as $g \rightarrow \infty$, where $\left|x_{i}^{c}-x_{j}^{c}\right| \rightarrow \infty$. The orbitals $u_{l}^{(i)}$ and the coefficients $\lambda_{l}^{(i)}$ are nothing but the eigenvectors (natural orbitals) and eigenvalues (occupancies) of $\Gamma^{g \rightarrow \infty}$, respectively. The values of $a_{i}, b_{i}$ and $A_{i}$ satisfy $a_{i}=a_{N-i+1}, b_{i}=b_{N-i+1}$ and $A_{i}=A_{N-i+1}$, which follows from the fact that the Hamiltonian (1) is invariant under the transformation $\vec{r} \rightarrow-\vec{r}$. As a result, if $N$ is even, all the asymptotic occupancies are twofold degenerate: $\lambda_{l}^{(i)}=\lambda_{l}^{(N-i+1)}$, whereas, if $N$ is odd, the asymptotic spectrum consists of occupancies that are twofold degenerate and those that are not degenerate: $\lambda_{l}^{\left(\frac{N+1}{2}\right)}$.

As a matter of fact, the above approach is semianalytical as in most cases the equilibrium positions of the particles have to be determined numerically. Nonetheless, the computational cost of such calculations is decidedly lower than that of full calculations. This provides us with a unique opportunity to gain insight into the properties of a Wigner molecule formed by a large $N$.

\section{Results}

Now we come to the point where we address the question of how the properties of the Wigner molecule ground-state depend on $N$ and $d$. It was concluded in [16] that, if $N$ and $d$ are relatively small, then, in the expansion (15), only the terms with $l=0$ are substantial; that is,

$$
\Gamma^{g \rightarrow \infty}\left(x, x^{\prime}\right) \approx \sum_{i=1}^{N} \lambda_{0}^{(i)} u_{0}^{(i)}(x) u_{0}^{(i)}\left(x^{\prime}\right)
$$

where $\lambda_{0}^{(i)} \approx N^{-1}$, which means that only the $N$ oneparticle states (natural orbitals) are significantly occupied. For example, for the system with $N=3, d=1$, we found: $\lambda_{0}^{(1)}=\lambda_{0}^{(3)} \approx 0.3249, \lambda_{0}^{(2)} \approx 0.3193$. However, the above issue has not yet been fully explored. Here we address it and reveal for which values of the control parameters of the system the formula (20) becomes valid. For the present purpose, it is convenient to use the so-called degree of correlation

$$
\mathrm{K}=P^{-1} \text {, }
$$

$P=\sum_{l} \lambda_{l}^{2}$, which counts approximately the number of natural orbitals actively involved in the expansion of the 1-RDM. This quantity is one of the transparent measures of correlation [36]. In the particular case that $\lambda_{i}=1 / N$, $(i=1, \ldots, N)$, it exactly gives the number of occupied one-particle states, $\mathrm{K}=N$.

In the perfect Wigner molecule regime as $g \rightarrow \infty$, in which $\lambda_{l}^{(i)}=\lambda_{l}^{(N-i+1)}$, the purity takes the form [15]

$$
\mathrm{P}^{g \rightarrow \infty}=2 \sum_{i=1}^{\frac{N}{2}} P_{i}
$$

and

$$
\mathrm{P}^{g \rightarrow \infty}=2 \sum_{i=1}^{\frac{N-1}{2}} P_{i}+P_{\frac{N+1}{2}}
$$

for even and odd values of $N$, respectively, $P_{i}=\sum_{l}\left(\lambda_{l}^{(i)}\right)^{2}$. As long as $\mathrm{K}^{g \rightarrow \infty}=\left(\mathrm{P}^{g \rightarrow \infty}\right)^{-1} \approx N$, the corresponding $1-\mathrm{RDM}$ is expected to have the property (20).

Figure 1 shows the dependence of $\mathrm{K}^{g \rightarrow \infty}$ on $N$ for different values of $d$, including the limit of $d \rightarrow 0$. In addition, to indicate deviations from (20), the values of $\mathrm{K}=N$ are 

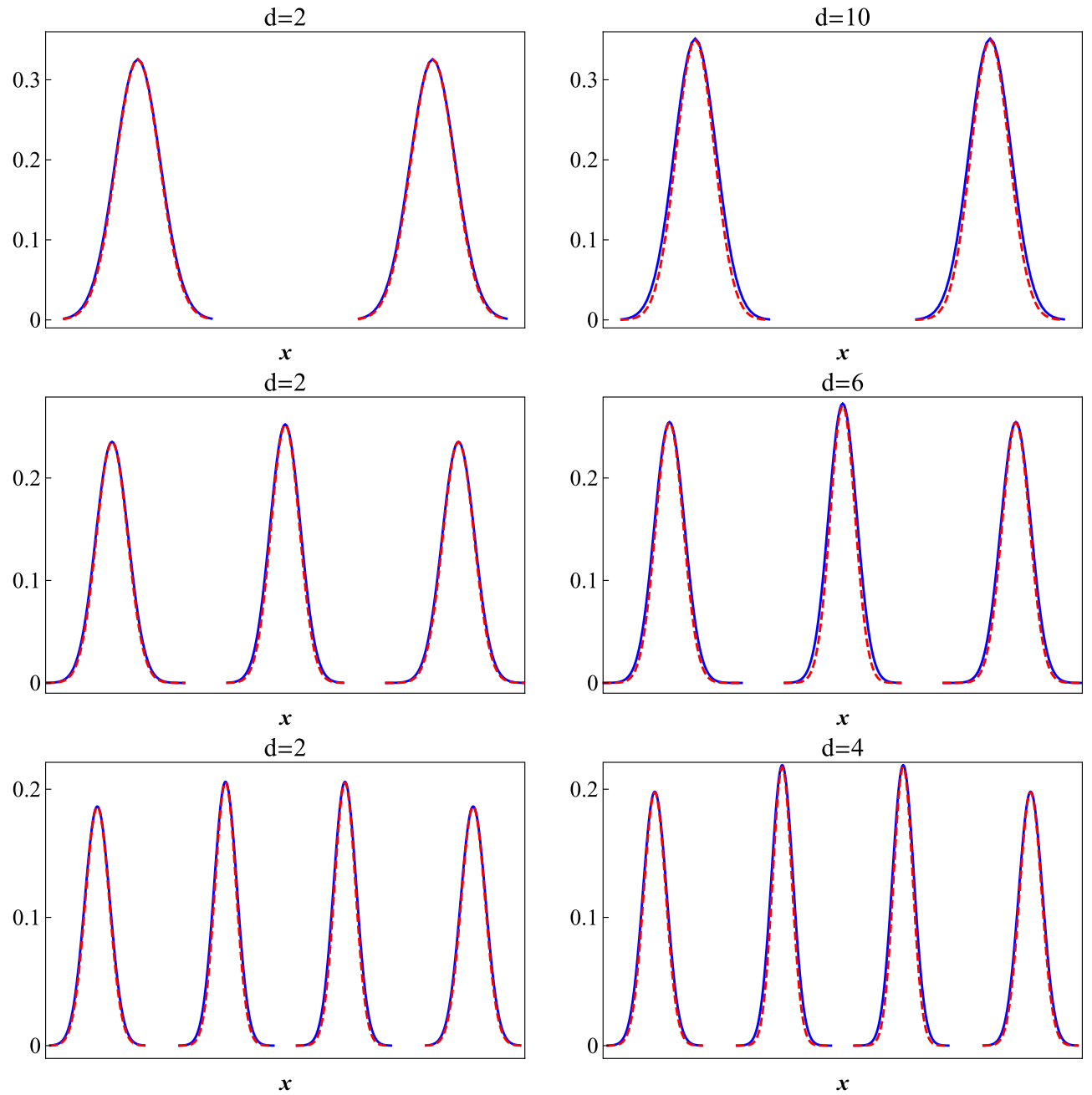

Fig. 2. Comparison of the one-particle density of a perfect Wigner molecule $(g \rightarrow \infty)$ ground-state with its approximation (20) for $N=2: d=2(\delta K=0.06), d=10(\delta K \approx 0.2) ; N=3: d=2(\delta K \approx 0.1), d=6(\delta K \approx 0.2)$ and $N=4: d=2(\delta K \approx 0.13)$, $d=4(\delta K \approx 0.2)$. For the sake of presentation, the density peaks are located at some fictitious points (in the limit as $g \rightarrow \infty$ the distance between the classical equilibrium positions of any pair of particles tends to infinity).

shown in this figure. It is apparent from the results that the value of $\mathrm{K}^{g \rightarrow \infty}$ deviates more and more from $N$ with the increase in $N$ and/or in $d$. This reflects the fact that, at the same time, the natural orbitals $u_{l}^{(i)}$ with $l$ higher than $l=0$, that is, these beyond the approximation (20), become increasingly important. We have estimated that the formula (20) yields a reasonable approximation (at least to the one-particle density) if the corresponding relative error $\delta K=\left(\mathrm{K}^{g \rightarrow \infty}-N\right) / N$ does not exceed a value of about 0.2 . Of course, the smaller is the value of $\delta K$, the better is the approximation (20). The above is proven by the results of Figure 2, which compares the density peaks of the perfect Wigner molecule (14)

$$
n_{i}(x)=\rho_{i}(x, x)=A_{i} \mathrm{e}^{c_{i}\left(x-x_{i}^{c}\right)^{2}}
$$

$c_{i}=-2 a_{i}+b_{i}$ with their one natural orbital approximations according to $(20), n_{i}(x) \approx \lambda_{0}^{(i)}\left[u_{0}^{(i)}\right]^{2}$. The results are presented for $N=2, \ldots, 4$ and some different values of $d$. As demonstrated here, the critical value of $d$ at which $\delta K=0.2$ decreases with increasing $N\left(N<N_{c r}\right.$, where $N_{c r}$ is the smallest value of $N$ at which $\delta K_{d \rightarrow 0}>0.2$ ).

Note that the two-particle Wigner molecule formed in the limit as $d \rightarrow 0$ is that for which the approximation (20) works the best. We obtained in this case $\lambda_{0}^{(1,2)}=2^{5 / 4} /\left(1+2^{1 / 4}\right)^{2} \approx 0.496, \mathrm{~K}^{g \rightarrow \infty} \approx 2.03$, which means that the sum of the remaining asymptotic occupancies is indeed vanishingly small: 0.007 . Interestingly enough, our inspection found that the partial components (14) tend to be within the limit as $d \rightarrow \infty$ to:

$$
\rho_{i}\left(x, x^{\prime}\right)=\frac{1}{\sqrt{\pi N}} \mathrm{e}^{-a_{i}\left(x-x^{\prime}\right)^{2}+c_{i}\left(x-x_{i}^{c}\right)\left(x^{\prime}-x_{i}^{c}\right)},
$$

with $a_{i} \rightarrow \infty$ and $c_{i}=-2 a_{i}+b_{i}=-N$. What follows from the above is that the corresponding 1-RDM $\Gamma^{g \rightarrow \infty}\left(x, x^{\prime}\right)$ does not exhibit contributions in the offdiagonal region (note that, if $x \neq x^{\prime}$, then $\mathrm{e}^{-a_{i}\left(x-x^{\prime}\right)^{2}} \rightarrow 0$ as $\left.a_{i} \rightarrow \infty\right)$. Accordingly, the corresponding one-particle 


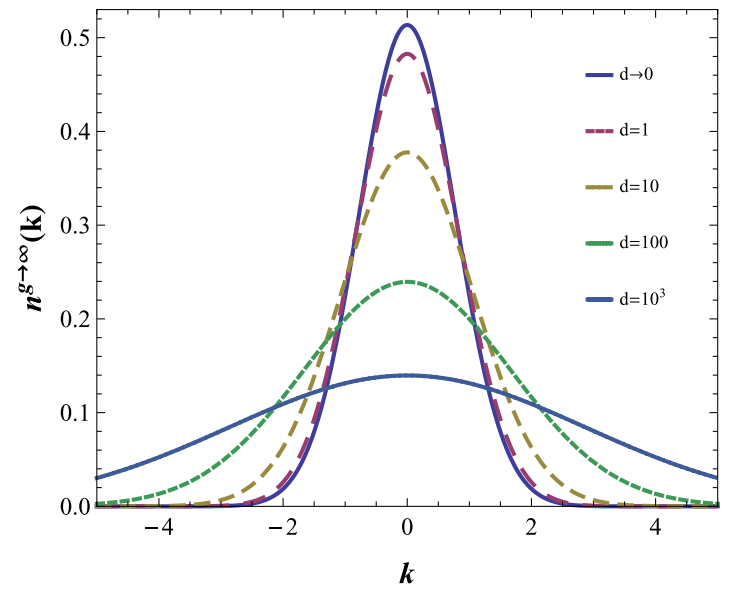

Fig. 3. The momentum distribution for the two-particle Wigner molecule ground-state as a function of $d$.

density peaks $\left(x=x^{\prime}\right)$ have the form

$$
n_{i}(x)=\frac{1}{\sqrt{\pi N}} \mathrm{e}^{-N\left(x-x_{i}^{c}\right)^{2}} .
$$

Further, bearing in mind equation (25), we immediately conclude that the values of $y_{i}$ in (19) tend to 1 as $d \rightarrow \infty$, which means that the clustering of the asymptotic occupancies (17) around zero occurs. In other words, in this limit, the infinite degeneracy occurs, namely the natural orbitals (16) correspond asymptotically to the same occupancy. Specifically, in the case of $N=2$, which can easily be tackled analytically, we found that all the occupancies $\lambda_{l}^{(1,2)}$ are asymptotically equivalent to $2(1 / d)^{1 / 4}$ as $d \rightarrow \infty, \lambda_{l}^{(1,2)} \sim 2(1 / d)^{1 / 4}$.

Despite the fact that the HA is valid only for finite values of $d$, one can expect that the Wigner molecule formed in the limit of sufficiently large $d$ will exhibit approximately the above properties.

Finally, we show in Figure 3 the results of how the momentum distribution

$$
\begin{aligned}
& n^{g \rightarrow \infty}(k) \\
& =\frac{1}{2 \pi} \int_{-\infty}^{\infty} \int_{-\infty}^{\infty} \Gamma^{g \rightarrow \infty}\left(x, x^{\prime}\right) \mathrm{e}^{-i k\left(x-x^{\prime}\right)} d x d x^{\prime},
\end{aligned}
$$

of the two-particle Wigner molecule ground-state changes with $d$. In this case, the integrals (27) can be performed explicitly:

$$
n^{g \rightarrow \infty}(k)=\frac{\sqrt{\frac{2}{\pi}} \mathrm{e}^{-\frac{2(\sqrt{d+2}-1) k^{2}}{d+1}}}{\sqrt{\sqrt{d+2}+1}} .
$$

As can be seen, the momentum distribution broadens as the value of $d$ increases, which reflects the fact that, at the same time, a reduction of density in the off-diagonal regions of $\Gamma^{g \rightarrow \infty}$ occurs. Obviously, for larger $N$, the similar behaviour is expected.

\section{Conclusions}

Within the framework of the HA, we studied the properties of the Wigner molecule formed by $N$ identical particles confined in a harmonic trap and interacting via the inverse power-law potential $|x|^{-d}$. Among other things, we elaborated the scheme to study the special case as $d$ approaches zero. Our results show the dependencies of the degree of correlation on $d$ for the $N$-particle Wigner molecule ground-state in a wide range of values of $N$. As a general trend we found that the number of natural orbitals contributing to the correlation of such a state grows with increasing $d$. In addition, we showed that the $1-\mathrm{RDM}$ of the Wigner molecule formed in the limit as $d$ approaches $\infty$ does not exhibit contributions in the offdiagonal regions. The corresponding one-particle density has been derived in a closed analytic form in the general case of $N$ particles. It turned out that it exhibits the peaks of the same profile.

Open Access This is an open access article distributed under the terms of the Creative Commons Attribution License (http://creativecommons.org/licenses/by/4.0), which permits unrestricted use, distribution, and reproduction in any medium, provided the original work is properly cited.

\section{References}

1. F. Tennie et al., Phys. Rev. A 93, 042126 (2016)

2. Y.C. Lin, C.Y. Lin, Y.K. Ho, Phys. Rev. A 87, 022316 (2013)

3. A. Majtey, A.R. Plastino, J. Dehesa, J. Phys. A: Math. Theor. 45, 115309 (2012)

4. C.L. Benavides-Riveros, I.V. Toranzo, J.S. Dehesa, J. Phys. B: At. Mol. Opt. Phys. 47, 195503 (2014)

5. D. Pȩcak et al., Phys. Rev. A 95, 053632 (2017)

6. C. Schilling, R. Schilling, Phys. Rev. A 93, 021601(R) (2016)

7. C. Schilling, Phys. Rev. A 88, 042105 (2013)

8. C.L. Benavides-Riveros, J.M. Gracia-Bondía, J.C. Várilly, Phys. Rev. A 86, 022525 (2012)

9. K.J.H. Giesbertz, R. van Leeuwen, J. Chem. Phys. 139, 104109 (2013)

10. T. Dornheim, A. Filinov, M. Bonitz, Phys. Rev. B 91, 054503 (2015)

11. A. Poszwa, Few-Body Syst. 57, 1127 (2016)

12. J. Dobrzyniecki, T. Sowiński, Eur. Phys. J. D 70, 83 (2016)

13. D. Pȩcak, T. Sowiński, Phys. Rev. A 94, 042118 (2016)

14. D. Pȩcak, M. Gajda, T. Sowiński, New J. Phys. 18, 013030 (2016)

15. P. Kościk, R. Maj, Few-Body Syst. 55, 1253 (2014)

16. P. Kościk, Phys. Lett. A 379, 293 (2015)

17. B. Szafran et al., Phys. Rev. B 69, 125344 (2004)

18. D.F.V. James, Appl. Phys. B 66, 181 (1998)

19. K. Balzer et al., J. Phys.: Conf. Ser. 35, 209 (2006)

20. M. Garagiola et al., Phys. Rev. A 94, 042115 (2016)

21. E. Cuestas et al., Phys. Lett. A 381, 2153 (2017)

22. O. Osenda et al., J. Phys. A: Math. Theor. 48, 485301 (2015)

23. F. Deuretzbacher et al., Phys. Rev. A 81, 063616 (2010) [Erratum: Phys. Rev. A 87, 039903 (2013)] 
24. N. Nielsen, I. Chuang, Quantum computation and quantum information (Cambridge University Press, Cambridge, 2000)

25. D. Wang et al., Phys. Rev. Lett. 93, 243005 (2004)

26. A.V. Gorshkov et al., IBID 101, 073201 (2008)

27. L. Jacak, P. Hawrylak, A. Wojs, Quantum dots (SpringerVerlag, Berlin, 1977)

28. N.T. Ziani et al., Phys. E: Low-Dimens. Syst. Nanostruct. 54, 295 (2013)

29. D.J. Wineland et al., Phys. Rev. Lett. 59, 2935 (1987)
30. E. Wigner, Phys. Rev. 46, 1002 (1934)

31. E.Y. Andrei et al., Phys. Rev. Lett. 60, 2765 (1998)

32. S. Kalliakos et al., Nat. Phys. 4, 467 (2008)

33. A. Singha et al., Phys. Rev. Lett. 104, 246802 (2010)

34. J.S. Meyer, K.A. Matveev, J. Phys. Condens. Mat. 21, $023203(2009)$

35. L. Guo, M. Liu, M. Marthaler, Phys. Rev. A 93, 053616 (2016)

36. R. Grobe, K. Rzążewski, J.H. Eberly, J. Phys. B 27, L503 (1994) 